\title{
MatML - Materials Markup Language Workshop Report
}

C.P. Sturrock

E.F. Begley

J.G. Kaufman 


\section{MatML - Materials Markup Language Workshop Report}

C.P. Sturrock

E.F. Begley

Materials Science and Engineering Laboratory

J.G. Kaufman

Kaufman Associates

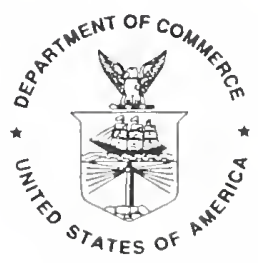

U.S. Department of Commerce Donald L. Evans, Secretary 



\section{TABLE OF CONTENTS}

Page

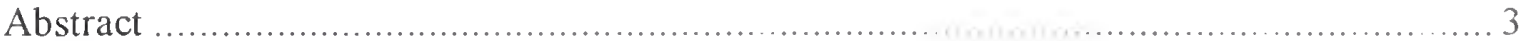

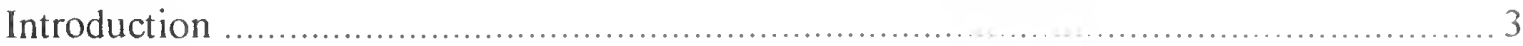

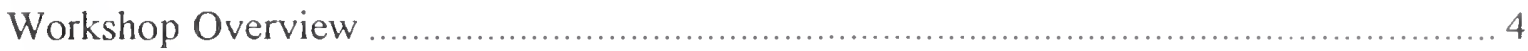

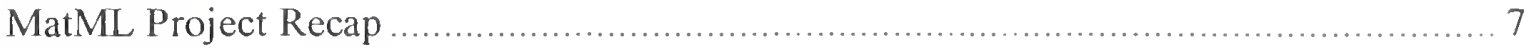

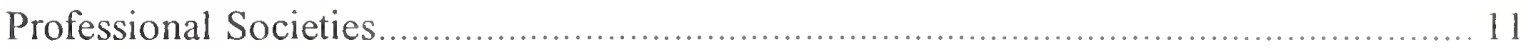

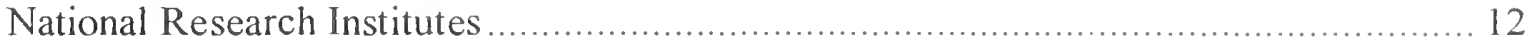

Academic Perspectives ...................................................................... 13

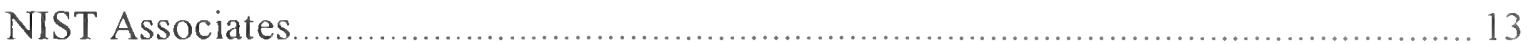

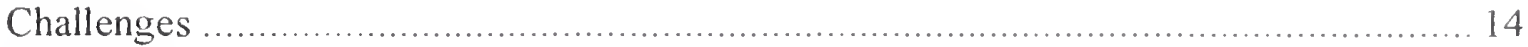

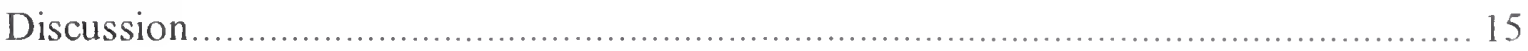

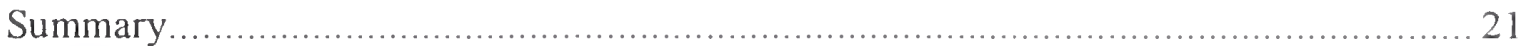

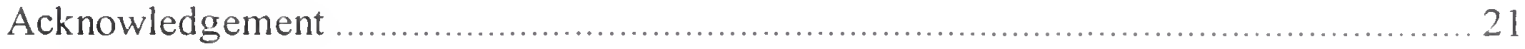

Appendices

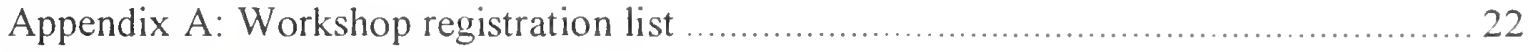

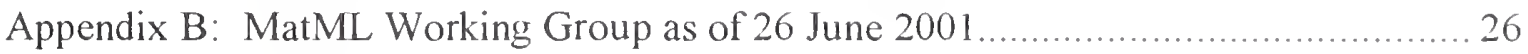

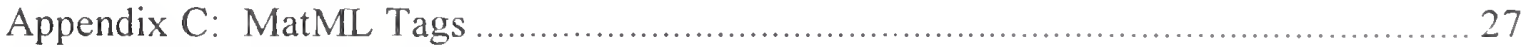

Appendix D: Sample Markup .............................................................. 28 



\section{MATML - MATERIALS MARKUP LANGUAGE WORKSHOP REPORT}

\section{ABSTRACT}

The MatML - Materials Markup Language Workshop was held at NIST to consider the technical and strategic future of MatML, an extensible markup language for materials data. Following detailed description and illustrations of MatML, the views of various constituencies of MatML were presented and considered. Several key strategic issues were identified, debated, and targeted for additional follow-up work. With the imminent migration of MatML from NIST to the broader user community, the longer-term stewardship of MatML and its potential formal standardization were recognized as the primary strategic issues to be resolved in the coming years.

\section{INTRODUCTION}

On June 26 and 27,2001, approximately 40 materials data experts (see Appendix A for complete list) convened at the National Institute of Standards and Technology to consider the future of MatML, an extensible markup language for materials data. The MatML Working Group, led by E.F. Begley of NIST, had developed MatML over the preceding 20 months via an online list server maintained at NIST. The workshop was co-chaired by Begley and C.P. Sturrock, also of NIST.

The widespread applicability of MatML attracted the participation of individuals from several types of organizations, including professional societies (e.g., ASM International, American Welding Society), national research institutes (e.g., the European Joint Research Center (Petten, The Netherlands), the National Institute for Materials Science (Japan), the U.K. Atomic Weapons Establishment), federally-funded data centers and agencies (e.g., the Advanced Materials \& Processes Technology Information Analysis Center, the National Aeronautics and Space Administration), manufacturers (e.g., RollsRoyce, Boston Scientific), software companies (e.g., MSC Software, Centor Corporation), universities, and materials consultants.

The goals of the workshop were threefold:

- Provide the MatML Working Group with a status report

- Introduce newcomers to MatML

- Develop consensus on MatML's technical and strategic future

The first day's format consisted of introductory presentations on related work at NIST, a brief overview of the workshop, an extensive multimedia presentation entitled: "MatML: Heritage, Music, Future", several panel sessions, and a challenge to the workshop attendees for the second day's work. The second day was devoted to recapitulation of the main points established on the first day, discussion of the MatML-related needs of the organizations represented, strategic issues, conclusions, and plans for future work. 


\section{WORKSHOP OVERVIEW}

\section{Welcome Address - S.J. Dapkunas, NIST}

NIST and NBS have been evaluating scientific data and developing scientific and technical databases for decades, dating back at least as early as the 1920s. Activities in recent years have evolved in several respects:

- The variety of materials and applications has continually expanded. The Structural Ceramics Database has been developed to address advanced heat engine needs and the NIST High-Temperature Superconducting Materials Database to serve the requirements of this very active scientific field.

- The method of product delivery has progressed from hard copy to computer disk to Web delivery. The Ceramics WebBook exemplifies this progression.

- The involvement and participation of the broad international technical community, including users and researchers has increased, as exemplified by this workshop.

- The development of mechanisms to more effectively use databases has become a component of the NIST ceramics data program.

These changes reflect both the opportunities made possible by affordable computers together with the rapid development of the World WideWeb; and, the changes in the user community:

- Today the commercial user community, often within a single company, is spread across the world and desires a greater variety of specific data on demand to meet the requirements of shorter product development times and cost effective manufacturing.

- The research community, also spread across the world within a given organization, creates data more rapidly through sophisticated modeling and experimental techniques such as combinatorial analysis.

MatML addresses these changing requirements of the research and development communities and it is important to carefully chart the future of this technology.

The Technical and Strategic Future of MatML - C.P. Sturrock, NIST

MatML has been under development since October 1999 by the MatML Working Group, established and led by E.F. Begley of NIST (see Appendix B for complete list of members). Motivated by the lack of a common materials data exchange format, and for the tremendous opportunities for data exchange and dissemination provided by the World 
Wide Web, MatML is an eXtensible Markup Language, based on the XML specification of the World Wide Web Consortium.

The differences between MatML and Hypertext Markup Language (HTML), the current lingua franca of the World Wide Web, can be seen plainly in the following example.

Table 1 contains data drawn from the NIST High-Temperature Superconducting Materials Database as it would appear when rendered by a web browser.

\begin{tabular}{|c|c|c|}
\hline Magnetic Field (T) & Temperature (K) & Critical Current Density (kA/cm2) \\
\hline 0 & 3 & 3040 \\
\hline
\end{tabular}

Table 1 - Data Excerpt from NIST High Temperature Superconductors Database

The HTML markup for this table is as follows, with the tags shown in italics and the data shown in normal text:

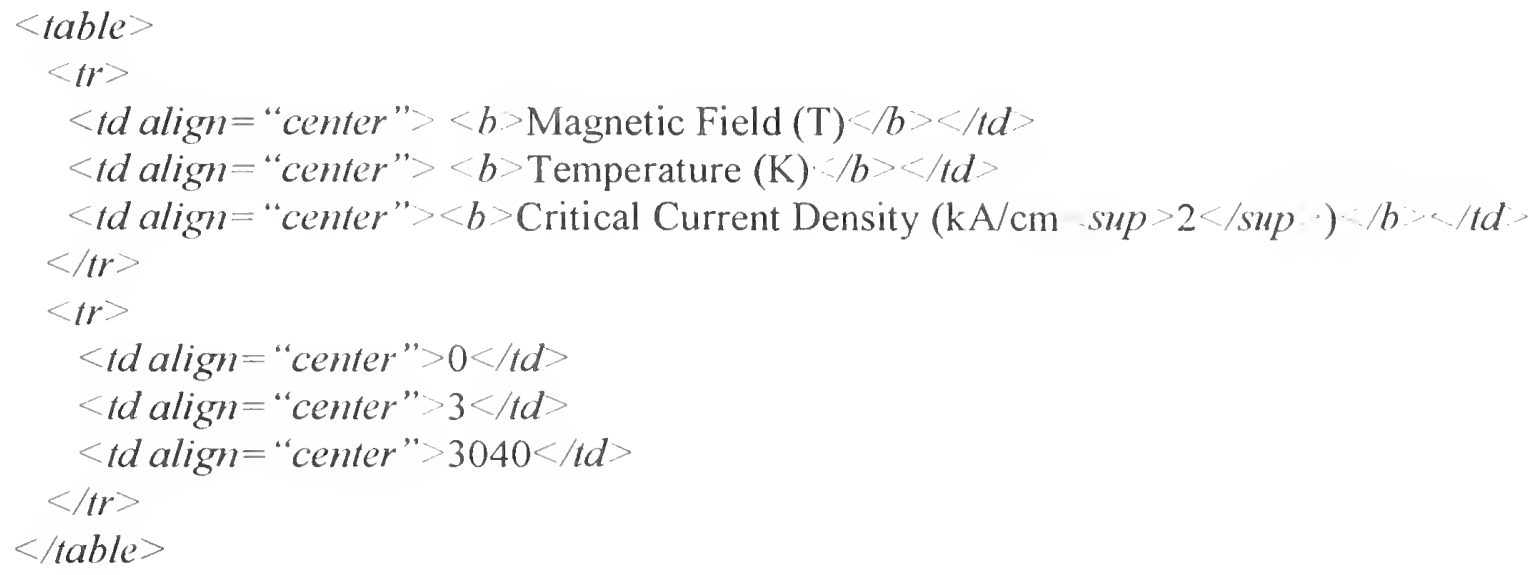

Note that the tags in HTML simply specify how the data are to be presented, and convey no descriptive content whatsoever.

Here is the same table in MatML markup:

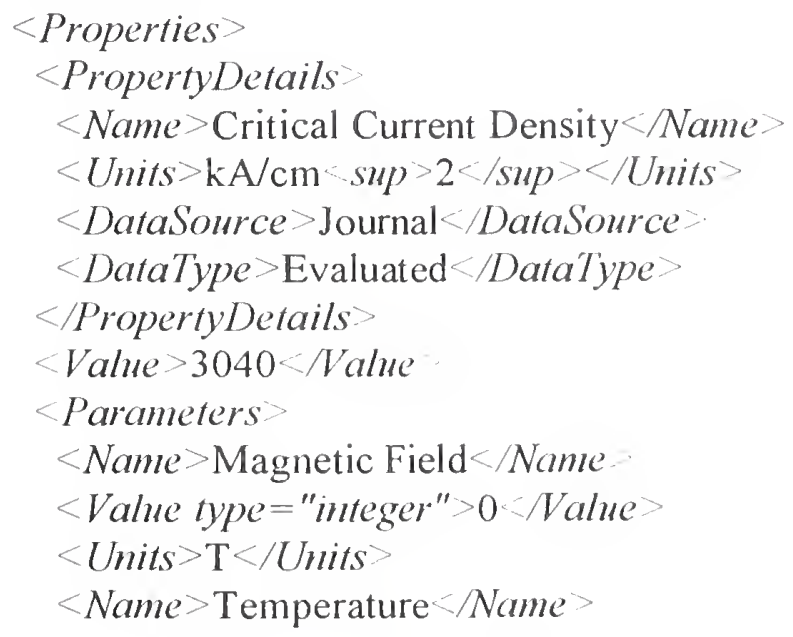




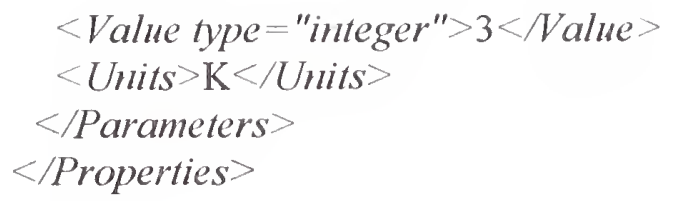

The descriptive nature of the MatML tags, such as $\langle$ Properties $\rangle,\langle$ Name $>$, and $\langle$ Units $\rangle$ is plainly evident. Note also that the MatML version includes other information about the pedigree of the data, as conveyed by $<$ DataSource $>$ and $<$ DataType $>$. MatML, like all extensible markup languages, provides for any number of user-defined tags such as these. The descriptive nature and extensibility of the language renders it far more intelligible and malleable than non-descriptive fixed tagsets such as HTML.

MatML has two primary lineages:

(1) general-purpose markup languages, e.g., Standard Generalized Markup Language, (SGML), HTML, XML, and

(2) materials data standards, e.g., those promulgated over the past two decades by the American Society for Testing and Materials (ASTM), the International Standards Organization (ISO), and other standards organizations.

Under development since October 1999, MatML Version 2.0 presently exists as a working draft in XML Document Type Definition (DTD) format. An annotated version of the entire DTD can be found via the MatML website:

http://www.ceramics.nist.gov/matml/matml.htm.

The future development and dissemination strategy for MatML includes the following steps:

- Develop catalog of examples,

- Test on examples and existing databases,

- Revise DTD as needed,

- Disseminate internationally.

Unlike MatML's development to date, which has proceeded in a linear fashion, the first two of these activities can be conducted in parallel. To reduce duplication of effort and maximize the effectiveness of parallel activities, it is strongly recommended that a coordinating body be established to oversee these efforts. It is further recommended that revisions (2.x) to MatML may be proposed at any time by anyone, but to be accepted, they must be approved by consensus of the MatML Working Group. 
MatML Project Recap: "The Heritage, Music, and Future of MatML" - E.F. Begley, NIST

\section{Heritage}

Various resources have been relevant to MatML's development. Of utmost significance were the discussions of the MatML Working Group that addressed the general specifications of simplicity, flexibility, and extensibility as well as the technical specifications, which are presently instantiated as the MatML DTD Working Draft Version 2.0. Other resources include: the Materials Information for Science and Technology (MIST) Project ${ }^{1}$, NIST Standard Reference Data Program materials databases, ASTM standards ${ }^{2}$, online as well as printed handbooks, manufacturer data sheets, e-journals, and ISO10303 Part 45 Integrated Generic Resource: Materials.

\section{$\underline{\text { Music }}$}

MatML, like music, exhibits simplicity in its notation, flexibility in its application, and extensibility in its range.

Simplicity is evident because:

1.) MatML uses plain text meaning that documents may be easily read, easily written, easily sent across a network, easily used by any programming language, and, consequently, support a wide variety of applications.

2.) MatML provides for straightforward structured documents composed of four primary types of information: a description of the bulk material, descriptions of the components that comprise the bulk material, descriptions of the material and property terms used in the document, and two-dimensional graphical images.

Each information type permits the use of an optional attribute, xmlns, for namespace linking to alterative content models.

3.) MatML defines tags that have meaningful names so the data are selfdescriptive (see Appendix C)

4.) MatML contains relatively few tags that, nonetheless, can be used to compose very complex documents.

Three fully worked examples of real materials data marked up using MatML are provided in Appendix D. These examples illustrate the flexibility of the language since they are for different materials from different publication sources:

- Structural Ceramic/Online Database - Source: "NIST WebSCD. http://www ceramics.nist.gov/srd/scd/Z00363.htm," R.G. Munro and E.F. Begley, 1998.

\footnotetext{
${ }^{\prime}$ W. Grattidge, J. Westbrook, J. McCarthy, C. Northrup, Jr., and J. Rumble, Jr., Materials Information for Science \& Technology (MIST): Project Overview, NBS Special Publication 726, Gaithersburg, MD, November 1986.

${ }^{2}$ ASTM Standards on the Building of Materials Databases, ASTM, Philadelphia, PA, 1993.
} 
- Aluminum Alloy/Handbook - Source: Generously provided by F. Cverna of ASM International and J.G. Kaufman of the Aluminum Association from Properties of Aluminum Alloys, p. 291, ASM International, Materials Park, Ohio, ISBN: 087170-632-6, 1999.

- Steel with TiC Coating/Journal - Source: A. Agarwal and N.B. Dahotre, "Pulsed Electrode Surfacing of Steel With TiC Coating: Microstructure and Wear Properties," ASM Journal of Materials Engineering and Performance, Vol. 8, No. 4, pp. 479-486, 1999.

Extensibility permits the MatML user to incorporate alternative data representations within the document markup and is accomplished through the use of namespaces.

Namespaces provide opportunities for extension and encourage reuse rather than reinvention. Some namespaces appropriate for use within a MatML document are:

- SVG - Scalable Vector Graphics (W3C)

- VHGTM - Virtual Hyperglossary (P. Murray-Rust, Virtual School of Molecular Sciences, UK)

- MathML - Mathematics Markup Language (W3C)

- CML - Chemical Markup Language (P. Murray-Rust, Virtual School of Molecular Sciences, UK)

- UnitsML - Measurement Units Markup Language (F. Olken and J. McCarthy, Lawrence Berkeley Labs and R. Dragoset, NIST)

- SpectroML - Molecular Spectrometry Markup Language (M. Rühl, M. Peschke, G. Kramer, NIST)

MatML can also be a namespace for other markup languages.

- femML - Finite Element Modeling Markup Language (J. Michopoulos, U.S. Naval Research Laboratory)

\section{Future}

The future of MatML will be shaped by responses to technical and strategic issues.

The three technical issues are the MatML DTD, acceptance testing, and education. With regard to the MatML DTD, MatML is not yet optimized. There is work still to be done concerning the materials content model and the XML representation of that model. Examples of changes to be made to the content model include providing support for components composed of components and providing support for the inclusion of images such as micrographs within a MatML document. Changes are also needed in the XML representation of the content model. These include the elimination of repetitive information, which became evident in the preparation of Appendix D, as well as migrating the DTD to an XML Schema representation given that XML Schema was released as a recommendation by the World Wide Web Consortium on 02 May 2001. XML Schema's provide robust data typing, including user-defined datatypes, and are 
much more suitable for application development than XML DTD's. Because the next phase of MatML's development will involve acceptance testing, the time is right for migrating to a MatML Schema in order to provide sound support for such testing.

With respect to acceptance testing, there are two principal kinds of XML applications: document publishing and document processing. Document publishing, which is the manipulation of information for human consumption, by necessity, comes first and will exhibit the strengths and weaknesses of MatML. In a sense, acceptance testing is already underway with the development of a compendium of markup examples but wider participation is needed in order to assess MatML's application to a broader and deeper range of materials. Future NIST testing might include markup of NIST WebSCD, NIST WebHTS, and NIST WebPDS, which are online compilations of structural ceramic, high temperature superconductor and property data summaries, respectively. ${ }^{3}$

Document processing, which is the manipulation of information intended for software consumption, represents the second phase of MatML's acceptance testing. While no specific plans have yet been identified, several possible projects are under consideration, including:

- Green's Function Research and Education Enhancement Network (Kent State, MIT, NIST)

- femML - the finite element modeling markup language (J. Michopoulos, U.S. Naval Research Laboratory)

The last technical issue is education. It is not sufficient to simply publish MatML as a language specification. It is necessary to provide additional resources to promote understanding and adoption of the new language. This philosophy was behind the publication of MatML in an annotated format where, in addition to the syntactic and semantic formalism of the language, English language explanations are provided as well as sample markup. Examples of other supporting resources for MatML include the MatML website, links to XML sources of information, and expositions such as "XML, Element Types, DTDs and All That. " Finally, the compendium of markup examples is extremely valuable for assisting users with learning how to apply MatML to their own data.

Presently the three main strategic issues for MatML are: promotion and outreach, MatML registration, and leadership and ownership.

Direct communications, journal publications, the MatML website, conference presentations, and the MatML workshop may be used to reach data providers and consumers such as journal editors, handbook and database publishers, materials' suppliers (data sheets), instrument manufacturers, and researchers. Registering MatML

\footnotetext{
${ }^{3}$ For more information, see the Ceramics WebBook at http://www.ceramics.nist.gov/webbook/webbook.htm

${ }^{4}$ For more information, see the MatML website at http://www.ceranics.nist.gov/matml/matml htm
} 
with the www.xml.org registry as well as the NIST registry/repository for XML may also provide broader exposure and access to MatML.

Lastly, MatML is now at a point in its development where new leadership focused on institutional issues, such as long term maintenance and support, is required. New leadership also requires expertise with pre-standardization protocols. It is recognized that the success of MatML requires continued NIST technical activity led by Begley as Chief Technical Expert focusing on:

- Continuation of the technical development and promotion of MatML.

- Continuation of participation in the MatML discussion forum.

- Development of an applications testbed for MatML acceptance testing - a necessary pre-standardization task.

- Providing technical assistance to anyone who wants to learn more about MatML and how to use it.

It was also recommended that:

- A neutral and international "umbrella organization" be identified or, if necessary, formed to:

- Manage the discussion forum

- Maintain the MatML DTD/Schema

- Organize acceptance testing activities

- NIST participate fully in the "umbrella organization"

- All MatML Working Group participants automatically become members and new members preserve diversity, i.e., represent a cross-section from industry, academia, government, professional societies, and standards organizations. 
Perspectives of various types of individuals and organizations were presented in panel discussions. The following sections summarize panelists' observations.

\section{PROFESSIONAL SOCIETIES}

\section{ASM International - F. Cverna}

While the MatML concept seems to be evolving nicely there are a number of issues not clear to ASM International about how they would use it and, most importantly, justify the expense of changing over their substantial data files into a MatML schema. In particular:

- A significant challenge seems to be how to test the MatML markup language

- Illustrations are needed of the relative value of MatML and traditional modes of exchange

- There seems some uncertainty on who will exchange data using MatML

- The relationship between a "document type definition" (DTD) and a schema needs some clarification

- Technical societies need more assurance that data quality and validation issues are properly handled by MatML

The Aluminum Association - J.G. (Gil) Kaufman (Ret.)

From the perspective of material scientists and engineers, the MatML concept is very attractive for two reasons: its extensibility, seemingly providing much more flexibility than existing protocol structures, and its understandability, the fact that because of the use of engineering materials terminology in the code, end user materials specialists and engineers can understand and evaluate the schema content and completeness. However, even assuming success and potential utility, some concerns remain:

- Usage - It is very easy to misunderstand the MatML concept as trying to create the ultimate database schema, rather than a markup language to facilitate schema development and data transfer for specific applications. The inclusion of illustrative applications in any documents on the subject will be helpful to material scientists and engineers in grasping the concept of MatML.

- Application to complex materials - Welded joints represent an example of what appears to be a challenge for MatML application not only because of the two components of the joint but also because of the need to be able to handle the transition region (i.e., the weld and heat-affected zones) adequately.

- Awareness - We will want to be careful that we do not repeat the situation where good technical development goes unknown and unused; at the appropriate time, a substantial publicity program including illustrative applications will be key.

- Understanding - While understandable in principle, MatML may easily be dismissed by those unwilling to take the time to digest it. Once again, the development of key illustrations of how specific schema are developed will be key. 
Thermal Spray Society - C. Berndt, State University of New York at Stony Brook

MatML has already and will continue to generate a positive impact on educators, researchers, academics, and journal editors, all of whom need to know how to handle large bodies of detailed, often numeric, data. Of particular interest, the requirements of the journal editor present significant challenges because of the need to gather on a continuing basis a variety of types of data for archival purposes while maintaining it readily accessible and searchable for many different types of users. The degree to which the use of MatML can be shown to have added value in saving time, space, or other cost elements will be key to editors decisions on its use.

CODATA - J.R. Rumble, Jr., NIST

Among the major concerns in the continued development and implementation of a concept like MatML is where the proper "home" for it should be, i.e., the umbrella under which further development, testing, evaluation, maintenance, and collaborative efforts might be managed. Among the potential homes that might be considered, CODATA has expressed an interest and willingness to oversee at least the technical aspects.

\section{NATIONAL RESEARCH INSTITUTES}

National Institute for Materials Science (Japan) - K. Halada

As of April 1, 2001, the National Research Institute for Metals and the National Institute for Research in Inorganic Materials were integrated into one organization: the National Institute for Materials Science (NIMS). The NIMS is engaged in several materials data pre-standardization projects, with partners elsewhere in Japan and abroad. In particular, the "NMC-MDB" effort, an application of XML to materials data, contains many parallels to the MatML effort.

Atomic Weapons Establishment (U.K.) - F. Moran

The Atomic Weapons Establishment (AWE) has the task of supplying data and datasheets to all AWE members. The AWE would like to improve the ability to search for and collect data available from other sources, and re-format it to their use, with the end result of improving material selection and design for their specific applications. Longer term, the goal is to facilitate the exchange and availability of materials data across networks. MatML shows promise in aiding with the AWE mission.

European Commission Joint Research Center (Petten, The Netherlands) - T. Austin

The EC's Joint Research Center in Petten would like to be in position to make their databases more accessible, including being searchable via the Internet. MatML could be useful for this purpose, especially if a data input module was available. Specific suggestions include: 
- Register MatML with some online registry organization;

- Establish regional/national repositories for schema developed with MatML, such as NIST, NIMS, JRC/IAM;

- Seek greater involvement of standards bodies (e.g., ASTM, ISO) in adopting specific materials schemas to go with each test standard.

\section{ACADEMIC PERSPECTIVES}

Kochi University of Technology - Y. Monma

Efforts are underway in Japan to compare XML-DTD in MatML and in NMC-MDB. Greater links between these markup language activities are desired, and efforts toward harmonization of recommendations being developed in Japan and the USA are encouraged.

Massachusetts Institute of Technology - A. Powell

In dealing with materials information in the academic environment, students should be encouraged to be skeptical of the quality and utility of data taken from various sources, and specifically taking the effort needed to enable the user to recognize the appropriate level of confidence that may be placed in the data. The potential usefulness of MatML is evident, and other educators are encouraged to teach the concept in their universities, especially its application for interactive programs to support math modeling.

University of Delaware - C. Newton

To be useful in the arena of advanced materials, MatML will need to readily handle composite materials, those typically made up of multiple materials and joining elements combined into an integrated structural material. One such example is a polymer-matrix composite material in which ten discrete elements can be identified. It is not obviously apparent that MatML can handle multiple properties from single specimens (though this is routinely done today with tensile test data, where tensile strength, yield strength, elongation, reduction of area, modulus and Poisson's ratio are often reported from single tests). Successful introduction of MatML, assuming its continued effective evolution, will involve giving it high visibility and dedicating substantial staff and resources to education and trial applications.

\section{NIST ASSOCIATES}

R.G. Munro, NIST Ceramics Division

The MatML project has reached a critical juncture. Up to this point, the focus has been on how to do it. The question, should it be done, was answered affirmatively early in the effort. Now that the initial DTD has arrived at the final stages of refinement, the question 
of how to get the word out has been raised. The answer is simply, show that it is useful. Once it is seen to be useful, the word of its availability will spread rapidly of its own accord. NIST perhaps will take a step in that direction by preparing the SCD (structural ceramics) and the HTS (high-temperature superconducting materials) databases in MatML. Beyond that, the major challenge will be to show that MatML is useful in an industrial or academic application. It is likely that this challenge will have to be addressed by someone outside of NIST.

\section{U.R. Kattner, NIST Metallurgy Division}

There is a question as to whether or not MatML will be able to handle relatively complex materials information such as phase transition diagrams and thermal equilibria. It is not yet clear what limitations will be encountered as the limits of applicability are tested and, assuming success, whether the effort to convert such massive compilations as the ASM/NIST phase diagram program to a MatML schema will be justified.

\section{S.J. Dapkunas, NIST Ceramics Division}

It is important to identify and involve in the implementation process those commercial interests who will most benefit from MatML's use. It will be far better if the implementation is "pulled" by those who need it rather than being seemingly "pushed" by the developers or technical advocates.

\section{Areskoug, Association of Swedish Engineering Industries (ASEI)}

A great opportunity exists within organizations such as ASEI to implement a markup language like MatML because of their need to link centers of knowledge with centers of industry and productivity. Through such organizations, the needs of small business as well as big business are effectively served.

\section{CHALLENGES}

\section{J.R. Rumble, Jr., NIST}

MatML is an important development at a time when it seems the need for data continues to increase, but in an environment where the activity to make it more readily accessible and of better quality seem to have diminished. Further implementation of MatML needs to be addressed with the following basic facts in mind:

- Information is a vital economic factor;

- Cost drives how information is moved;

- There seems to be limited opportunity for added revenue from materials information.

In spite of the best materials data standards efforts to date, such standards have had very small impact on materials databases. Virtually none of the materials data resources developed over the past two decades work together. Moving data from them into 
application software is still as difficult as ever. Despite millions of dollars of investment, the Standard for the Exchange of Product Data (STEP) has not produced any real impact on materials databases or software that might use them. By contrast, MatML offers considerable promise because it is easy to understand, easy to use and easy to improve.

Doing nothing does not seem to be an option at this point, but rather the combined input of all those with an interest in MatML is needed to:

- Identify the business reasons for adopting MatML;

- Identify sample applications that may be the best business opportunities to implement MatML;

- Identify the means of focusing the effort while assuring globalization of the application to the degree possible.

Possible organizations to consider approaching for assistance with pre-standardization and/or standardization efforts include: the Versailles Project on Advanced Materials and Standards (VAMAS), the American Society for Testing and Materials (ASTM), the International Standards Organization (ISO), the Committee on Data for Science and Technology (CODATA), and the International Union of Materials Societies (IUMS).

\section{DISCUSSION}

The observations that follow are the consolidated summary of the input from participants throughout the workshop, presented in four sections:

- Technical Issues - How is MatML doing? What remaining technical issues need to be addressed?

- Strategic Issues - Where should MatML go from here?

- Organizational Issues - How do we get there?

- Identification of current and needed participants

\section{Technical Issues}

From the panel presentations and the resulting discussion, it was clear that the development of MatML to date is meeting with great interest and, generally, considerable optimism. There were at least three reasons noted for this interest and optimism:

- The high level of effort and care with which the development has proceeded to date under the leadership of E.F. Begley and the team of specialists he has assembled to critically evaluate and provide input to the development of the basic markup language and the DTD,

- The obvious extensibility of the markup language, making it potentially very easily and efficiently expanded to be applicable to the full range of materials information needed by materials researchers, specialists, designers, and quality control analysts, and to the full range of applications for which MatML would be utilized to develop specific schema, and 
- The relative ease of understanding the code (control symbols) utilized to design and implement specific schema; basically the code consists of materials and engineering terminology, which has the key advantage of enabling materials scientists and engineers to review the schema in detail to ensure that all of the required parameters involving test methods, quality, reliability, and applicability of the data have been addressed. Other markup languages developed to date do not provide this important advantage.

Despite the recognition at various levels of these desirable features, there remain a number of technical issues that must be addressed and/or demonstrated.

Most of the questions about MatML fall into four major categories:

- How well will data encoded and transferred in MatML-based schema be able to be searched by a variety of different individuals with a wide variety of missions in mind, whether alloy selection, design, or quality assurance? Will the complexities of materials terminology be able to be dealt with (e.g., Young's modulus vs. modulus of elasticity in tension)?

- How will MatML handle complex materials? Will it be practical (as contrasted to possible) to define the properties and characteristics of multicomponent polymer-, ceramic-, or metal-matrix composites, some of which (including the contact conditions) may represent up to ten individual elements? Will it be possible to adequately describe joints, such as welds in metallic materials where there are typically two primary components and a transition region where properties vary with location (i.e., the heat-affected zone)?

- Will MatML be able to handle more complex materials information such as phase diagrams, heat transfer data, chemical reactions, and graphical or pictorial data such as microstructures?

- Is there adequate opportunity within MatML to address descriptors of data quality and reliability, including the statistical reliability (ranging from individual test data and averages to various calculated confidence limits) and detail on test methods (whether they were standard methods, some variations of standard methods, or non-standard procedures)?

While not all of the various situations raised in these questions have been illustrated and tested critically, the very nature of the flexibility and extensibility of MatML does not preclude any of these types of information being handled effectively. In many cases, the functionality would be guided and the success determined by the specific schema generated to handle the application and types of data involved, such as for composites, as illustrated in Appendix D, Example 3. 
Finally, the development of a MatML editor would greatly facilitate the preparation of MatML documents.

\section{$\underline{\text { Strategic Issues }}$}

In order to effectively address the strategic issues of where the development of MatML should go from its present stage, it will be necessary to:

- "... demonstrate a business reason for MatML"

- "....make it (i.e., MatML) work for the materials community".

Economics is clearly at the heart of addressing these issues. In order to establish business reasons for MatML, there is a need to demonstrate that it can enable a business to:

- Increase sales of an existing product,

- More effectively introduce a new product,

- Increase the productivity of operations, or

- Reduce cost in some other manner.

Recognizing these factors, it is possible to identify some of the strategies needed for MatML to move forward, notably:

- Establish a good set of illustrations, emphasizing those that demonstrate the power and flexibility of MatML, and those with potentially significant business impact.

- Test MatML thoroughly, involving individuals at various stages in the material development/application cycle ranging from material specialists who do alloy selection to designers responsible for addressing load-carrying capacity and failure limits. The needs of research engineers need to be included and, on the opposite extreme, the needs of the journal publishers who must report, archive, and make searchable their materials information.

- Do what is necessary to have MatML recognized officially/formally. External recognition will clearly add validity to MatML. Both relatively informal but influential organizations like OASIS, or the more formal Object Management Group (OMG), should be considered. Standardization via ASTM is a possibility, and there is some precedent for this as ISO has included STEP-XML approaches in its "product description standards" for computerized systems. ASTM Committee E-49, though now inactive, was responsible for the development of about 30 standards for computer descriptions of materials and test results, and might be a logical home for efforts to have MatML recognized as a standard.

- Have a "reputable" home for the MatML markup language and related schema. In this context, "reputable" is intended to mean exceptionally high and widely recognized technical strength and integrity, sufficient to speak for the United 
States in international technical arenas such as CODATA, VAMAS, and ISO, and potentially to coordinate and/or lead the international effort to evaluate and if appropriate implement MatML. NIST leadership position in the work to date and its technical standing combined with its relative freedom from commercial conflicts of interest make it the logical place for the effort to continue. In addition, NIST would be a very logical place for a repository of MatML-based code as the applications emerge.

- Establish links with key industries that might, via some of their applications, be in position to provide good tests for MatML and, assuming success, encourage or perhaps even require users of their information systems to use MatML as the data transfer medium. Examples include:

- Include MatML implementation in the ASTM data standards created by ASTM Committee E-49;

- Encourage testing machine companies (e.g., MTS, Instron, Tinius Olsen) to build MatML markup language into their testing machine data recording/reporting systems;

- Encourage design protocol developers (e.g., MSC Software, formerly McNeal-Schwendler Corporation) to build MatML markup language into their software systems;

- Encourage materials software developers and distributors (e.g., Centor Corporation, ESM Software, knovel Corporation) to markup and disseminate their materials data products using MatML;

- Work with the purchasing departments of large industrial companies (especially automotive, aerospace) to require of their vendors to supply/receive materials information, including price, with MatML-based schema.

\section{Organizational Issues}

Having identified some technical and strategic issues along with strategies for addressing these issues, the next challenge is to identify how and who should implement these strategies. The organizational strategy can itself be thought of in two parts, and the potential candidates for implementation of those components of the strategy are identified in the following sections.

\section{(1) Technical Oversight}

It has already been noted that the technical leadership has come from and probably should logically continue to come from NIST. However, it is well to consider the important international contributions already being made to the basic problem by scientists such as Drs. Halada and Monma in Japan as well as the potential opportunity for greater input and testing from the broader international community. This could be accomplished via CODATA, the Committee on Scientific and Technical Data of the International Council of Scientific Unions; CODATA is a widely recognized and respected international organization dealing with a very wide range of scientific and 
technical data. CODATA has a history of activity in this area through its Task Group on Materials Database Management. VAMAS and ISO are other well-respected organizations that might be considered, though their scopes are somewhat narrower with regard to breadth of scientific data.

One possible alternative for broader international recognition would be to develop a proposal for a CODATA Task Group Activity that would encourage the international community to become involved in the further development and testing of MatML and the harmonization with other approaches to the use and/or modification of XML DTD for materials data.

\section{(2) Standardization/Registration}

Business considerations provide the incentive for having MatML registered or standardized by an appropriate body. Candidate organizations for registration and/or standardization include:

- OASIS/W3C;

- OMG (Object Management Group);

- ANSI/ASTM (ASTM is the major test methods standards writer for the American National Standards Institute, ANSI);

- ISO (International Standards Organization);

- IUMS (International Union of Materials Societies).

OASIS and/or OMG are the most logical candidate organizations for online registration, and ASTM the most logical if full standardization is desired; contact with these organizations to explore the opportunities and limitations should be initiated.

\section{$\underline{\text { Identification of Current and Needed Participants }}$}

It is useful both in implementing strategy and in addressing the needs identified and discussed above to note the existing participants (meaning in this case, those already aware of or in position to evaluate their interest in MatML technology) and those who will or may be needed in the future and might be contacted relatively soon to get involved. A significant measure of level of involvement at this stage will be considered to be participation in this workshop. In the context used at this time, the attention is upon organizational involvement (as contrasted to individual personnel).

(1) Present Participants (directly or indirectly, in no particular sequence)

- ASEI (Sweden; Areskoug)

- EU-JRC (Austin)

- NIMS (Japan; Halada, Monma)

- NIST (Sturrock, Begley)

- ASM International (Cverna)

- University of Delaware (Newton)

- MSC/McNeal-Schwendler (Kipp)

- Centor (Nunez)

- Knovel (Gurke [not represented]) 
- NASA MSFC (Mitchell)

- NCMS (indirect; Oakwood)

- Boston Scientific (Doyle)

- AISI (indirect; Oakwood)

- Aluminum Association (indirect; Kaufman)

- ASTM (indirect; Newton)

- Granta (indirect; Cverna)

- Ford (indirect; Nunez)

(2) Desirable Additional Participants

- Testing machine companies (e.g., MTS, Instron, Tinius Olsen)

- Engineering software companies (e.g., Parametrics, Unigraphics)

- ASTM (via its management, not individual committees)

- Other materials-heavy universities (e.g., VA, PA)

- DOE laboratories (e.g., ORNL, Sandia, LLL)

- Other automotive companies and USCAR

- Business associations in energy (e.g., AGA, API)

- Institute of Metals (UK)

- Insurance companies

- Medical community

- Energy companies (e.g., Exxon-Mobil, BP-ARCO)

- Managers of related websites (e.g., MATWEB)

To engage these potential participants effectively in further development and testing of MatML, separate leadership will be required, apart from technical leadership. A MatML Steering Committee, much smaller in size than the full Working Group, will be better suited to future tasks such as building additional user interest, promoting adoption of MatML, coordinating testing, engaging other organizations and individuals in prestandardization efforts, etc. Several individuals present at the workshop expressed interest in exploring contacts on behalf of MatML, in providing sample MatML markup or data for others to mark up, and/or in serving on the MatML Steering Committee. 


\section{SUMMARY}

A workshop was held to consider the technical and strategic future of MatML, a markup language for materials data. Perspectives were obtained from representatives of the automotive, scientific instrument, and software industries, from materials-related professional societies, from national research institutes, from federally funded scientific data centers and agencies, from academia, and from various materials consultants.

The development of MatML to date is meeting with great interest and, generally, considerable optimism. MatML has clearly reached the stage where testing and sample development are needed to demonstrate its utility and promote its use. Several workshop attendees agreed to contribute samples of MatML markup based on their data, as well as sample data for markup and testing by others. It was observed that a MatML editor would greatly facilitate the preparation of MatML documents. A number of strategic issues remain to be resolved:

- The overall coordination of testing and sample development;

- Registration of MatML at a recognized on-line repository;

- A permanent home for the longer-term access to and maintenance of MatML.

A Steering Committee will be established in the coming months to address these issues.

\section{ACKNOWLEDGEMENT}

The authors acknowledge the valuable editorial suggestions of S.J. Dapkunas in the preparation of this report. 
Appendices

Appendix A: Workshop registration list

Magnus Areskoug

Association of Swedish Engineering

Industry

Box 5510

Stockholm SE-1 1485, SWEDEN

Telephone: 46/8782054

Fax: 46/87820900

Email:magnus.areskoug@vi.se

Tim Austin

European Commission DG-JRC

Westerduinweg 3

Petten, NH 1755-ZG NETHERLANDS

Telephone: $31 / 224565203$

Fax: $31 / 22456522$

Email: austin@jrc.nl

Laura Bartolo

Kent State Univ.

161 University Library

Kent, OH 44242 USA

Telephone: 330/672-1691

Fax: 330/672-3964

Email: lbartolo@kent.edu

Ed Begley

NIST

100 Bureau Dr., Mail Stop 8520

Gaithersburg, MD 20899-8520 USA

Telephone: 301/975-6118

Fax: 301/975-5334

Email: edwin.begley@nist.gov

Alec Belsky

NIST

100 Bureau Dr., Mail Stop 8520

Gaithersburg, MD 20899-5334 USA

Telephone: 301/975-8325

Fax: 301/975-5334

Email: alec.belsky@nist.gov

Christopher Berndt

SUNY at Stoney Brook

306 Old Engineering

Stony Brook, NY 1 1794-8525 USA

Telephone: 516/632-8507

Fax: 516/632-8525

Email: cberndt@notes.cc.sunysb.edu

Fran Cverna

ASM International

9639 Kinsman Rd.

Materials Park, OH 44073 USA

Telephone: 440/338-5151

Fax: 440/338-4634

Email: fcverma@asminternational.org

Sandy Dapkunas

NIST

100 Bureau Dr., Mail Stop 8520

Gaithersburg, MD 20899-8520 USA

Telephone: 301/975-6119

Fax: 301/975-5334

Email: stanley.dapkunas@nist.gov

Sarah Doyle

Boston Scientific Corp.

One Boston Scientific Place

Natick, MA 01760 USA

Telephone: 508/652-5255

Fax: 508/5650-8936

Email: doyles1@bsci.com

Marc El-Khoury

Johns Hopkins Univ.

3400 N. Charles St.

206 Latrobe Hall

Baltimore, MD 21218 USA

Telephone: 410/516-0068

Fax: 410/516-7473

Email:marckhoury@jhu.edu 
Marcelle Gaune-Escard

IUSTI

5, rue Enrico Fermi

Marseille 13453, FRANCE

Telephone: 33/4 91106887

Fax: 33/491117439

Email:mge@iusti.univ-mrs.fr

David Goodall

Rolls-Royce Plc

P.O. Box 31

Mail Code ELT-11

Derby DE24 8BJ, UNITED KINGDOM

Telephone: 44/1332 240467

Fax: 44/1332 241081

Email: david.goodall@rolls-royce.com

Benjamin Grenier

Embassy of France

4101 Reservoir Rd., NW

Washington, DC 20007 USA

Telephone: 202/944-6241

Fax: 202/944-6244

Chris Grethlein

AMPTIAC

201 Mill St.

Rome, NY 13440 USA

Telephone: 315/339-7009

Fax: 315/339-7107

Email: cgrethlein@iitri.org

Kohmei Halada

Natl. Inst. for Materials Science

1-2-1 Senger Tsukuku

3050047, JAPAN

Telephone: 81/298 592352

Fax: 81/298-59-2301

Email: halada.kohmei@nims.go.jp
Vicky Lynn Karen

NIST

100 Bureau Dr., Mail Stop 8520

Gaithersburg, MD 20899-8520 USA

Telephone: 301/975-6255

Fax: 301/975-5334

Email: vicki.karen@nist.gov

Ursula Kattner

NIST

100 Bureau Dr., Mail Stop 8555

Gaithersburg, MD 20899-8555 USA

Telephone: 301/975-6044

Fax: 301/975-4553

Email: ursula.kattner@nist.gov

J.G. Kaufman

Kaufman Associates

3662 Pevensey Dr.

Columbus, OH 43220-4821 USA

Telephone: 614/459-3949

Fax: 614/459-3949

Email: gil.kaufman@worldnet.att.net

Tom Kipp

MSC Software

2975 Redhill Ave.

Costa Mesa, CA 92626 USA

Telephone: 714/444-5060

Fax: 714/979-2990

Email: tom.kipp@mscsoftware.com

Michael Mitchell

NASA-MSFC

ED35

Huntsville, AL 35812 USA

Telephone: 256/544-2491

Fax: 256/544-5478

Email:michael.mitchell@msfc.nasa.gov

Kevin Heath

Boston Scientific

1 Boston Scientific PI.

Natick, MA 01760 USA

Telephone: 617/972-4271

Email: heathk@bsci.com 
Yoshio Monma

Kochi Univ. of Technology

Tosayamada-cho

Kami-gun

Kochi 782-8502, JAPAN

Telephone: 81/88757 2515

Fax: 81/887 572520

Email:monma@env.kochi-tech.ac.jp

Frederick Moran

Atomic Weapons Establishment

Bldg. C4

Reading R47 4PR, UNITED

KINGDOM

Telephone: 44/1189826690

Email: fred.moran@awe.co.uk

Ronald Munro

NIST

100 Bureau Dr., Mail Stop 8520

Gaithersburg, MD 20899-8520 USA

Telephone: 301/975-6127

Fax: 301/975-5334

Email: ronald.munro@nist.gov

Thomas Mustaleski

American Welding Society

P.O. Box 2009

Oak Ridge, TN 37831-8096 USA

Telephone: 865/574-1820

Fax: 865/574-2582

Email:tmm@y12.doe.gov

Crystal Newton

Univ. of Delaware

Center for Composite Materials

201 CMSL

Newark, DE 19716 USA

Telephone: 302/831-1017

Fax: 302/831-8525

Email: newton@ccm.udel.edu
Chris Nunez

Centor Software Corp.

4 Park Plaza, Ste. 900

Irvine, CA 92614 USA

Telephone: 949/757-2304

Fax: 949-757-2311

Email: cen@center.com

Thomas Oakwood

Metallurgical Engineering Consultant

701 Brandonbury Dr.

Valparaiso, IN 46383 USA

Telephone: 219/462-5235

Fax: 219/462-5235

Email: oakwoodt@wordlnet.att.net

Mark Patterson

Tech. Assessment \& Transfer, Inc.

133 Defense Hgwy., Ste. 212

Annapolis, MD 21401 USA

Telephone: 301/261-8373

Fax: 410/224-4678

Email: info@techassess.com

Adam Powell

Massachusetts Institute of Technology

Rm. 4-117

Cambridge, MA 02139 USA

Telephone: 617/451-2086

Fax: 617/253-5418

Email: hazelsct@mit.edu

Shrirang Ranade

Boston Scientific

1 Boston Scientific

Natick, MA 01760 USA

Telephone: 508/652-5255

Email: doyles1@bsci.com

Manfred Roth

EMPA

Dubendorf 8600, SWITZERLAND

Email:manfred.roth@empa.ch 
Ranganath Shastri

Dow Chemical Co.

2040 Dow Center

Midland, MI 48674 USA

Telephone: 989/636-9034

Fax: 989/638-9845

Email: rshastri@dow.com

Charles Sturrock

NIST

100 Bureau Dr., Mail Stop 2310

Gaithersburg, MD 20899-2310 USA

Telephone: 301/231-6027

Fax: 301/926-0416

Email: charles.sturrock@nist.gov
Jack Westbrook

Brookline Technologies

5 Brookline Rd

Ballston Spa, NY 12020 USA

Telephone: $518 / 885-8840$

Fax: 518/885-8840

Email:jwestbrook@attglobal.net 
Appendix B: MatML Working Group as of 26 June 2001

\section{NIST members}

E. Begley, Principal Investigator and Project Leader

B. Boettinger

S. Dapkunas

S. Freiman

C. Handwerker

V. Karen

U. Kattner

R. Munro

J. Rumble

T. Siewert

C. Sturrock

C. White

\section{Non-NIST members}

T. Baba, National Research Laboratory of Metrology, Japan

C. Bullough, ABB ALSTOM Power, Leicester UK

C. Gibson, CINDAS, Purdue University

S. Gurke, William Andrew Publishing

K. Halada, National Research Institute for Metals, Japan

G. Kaufman, Aluminum Association, Inc.

T. Kipp, MSC. Software

D. Martin, Iowa State University

J. McCarthy, Lawrence Berkeley National Laboratory

S. McCormick, ESM Software

J. Michopoulos, U.S. Naval Research Laboratory

F. Moran, Atomic Weapons Establishment, UK

P. Murray-Rust, Nottingham University, UK

C. Newton, University of Delaware

F. Olken, Lawrence Berkeley National Laboratory

J. Phipps, William Andrew Publishing

A. Powell, Massachusetts Institute of Technology

D. Readey, Colorado School of Mines

D. Rose, AMPTIAC

M. Smith, CenTOR Software Corporation

M. Stoeckle, Ford Motor Company

M. Sullentrup, The Boeing Company 
Appendix C: MatML Tags

\begin{tabular}{|l|l|l|}
\hline Associate & Elements & Processing \\
\hline Associations & Form & Properties \\
\hline BulkDetails & Formula & PropertyDetails \\
\hline Characterization & Geometry & Qualifier \\
\hline ChemicalComposition & Graphs & Relationship \\
\hline Class & Material & Result \\
\hline Component Details & MatML_Doc & Shape \\
\hline Compound & Measurement Technique & Source \\
\hline Concentration & Name & Specification \\
\hline DataSource & Notes & Subclass \\
\hline DataType & Orientation & Terms \\
\hline DimensionDetails & Parameters & Units \\
\hline Dimensions & PhaseComposition & Value \\
\hline
\end{tabular}




\section{Appendix D: Sample Markup}

Please note that these examples illustrate the flexibility of MatML even if MatML is not yet optimized. The purposes of these examples are to provide informative illustrations for potential users of MatML and to uncover the strengths and weaknesses of the MatML Working Draft in order to formulate a plan for optimizing MatML and moving it forward to proposed recommendation status. (Example: Notice the information redundancy in the markup that must be addressed in a future version of MatML.)

The reader interested in the markup of complex materials systems such as composites is referred to Example 3.

Example 1: Structural ceramic from an online materials database

Source: "NIST WebSCD: http://www.ceramics.nist.gov/srd/scd/Z00363.htm," R.G. Munro and E.F. Begley, 1998.

$<$ ? xml version="1.0"? $>$

$<$ !--DOCTYPE MatML_Doc SYSTEM "MatMLv20.dtd"-->

$<$ MatML_Doc $>$

$<$ Material $>$

$<$ BulkDetails $>$

$<$ Name $>$ silicon nitride $<$ Name $>$

$<$ Class $>$ ceramic $</$ Class $>$

$<$ Specification $>$ NCX-5 $102</$ Specification $>$

$<$ Source $>$ Saint-Gobain/Norton Industrial Ceramics

$<$ Form $>$ bar $<$ Form $>$

$<$ Processing $>$

$<$ Name $>$ hot isostatic pressing $<$ Name $>$

$<$ Notes $>$

"The material produced is designated NCX-5102 and consists of a silicon nitride-4\% yttria composition that is densified by glass-encapsulation HIPing. ... Large-scale batches $(30 \mathrm{~kg})$ of $\mathrm{Si}<$ sub $>3</$ sub $>\mathrm{N}<$ sub $>4</$ sub $>-4 \% \mathrm{Y}<$ sub $>2</$ sub $>0<$ sub $>3</$ sub $>$ powder were milled in water,

and the slurry was used to cast hundreds of tensile rods. The starting

$\mathrm{Si}<$ sub $>3</$ sub $>\mathrm{N}<$ sub $>4</$ sub $>$ powder (Ube) was derived from a dimmide process. ... The netshape-formed bars were HIPed using glass encapsulation (ASEA Cerma AB, Robertsford, Sweden). The HIP process was optimized using pressure, time and temperatures to assure full densification and development of an elongated microstructure for desired fracture toughness..."

$<$ Notes $>$

$</$ Processing $>$

$<$ Characterization $>$

$<$ Formula $>$

Si $<$ sub $>3</$ sub $>$ N $<$ sub $>4</$ sub $>\& \# 183 ; 4$ wt $\% \mathrm{Y}<$ sub $>2</$ sub $>0<$ sub $>3</$ sub $></$ Formula $>$

$<$ ChemicalComposition $>$

$<$ Compound $>$

$<$ Element $>$ Si $</$ Element $>$

$<$ Units $>3</$ Units $>$

$<$ Element $>\mathrm{N}</$ Element $>$

$<$ Units $>4</$ Units $>$

$</$ Compound $>$ 


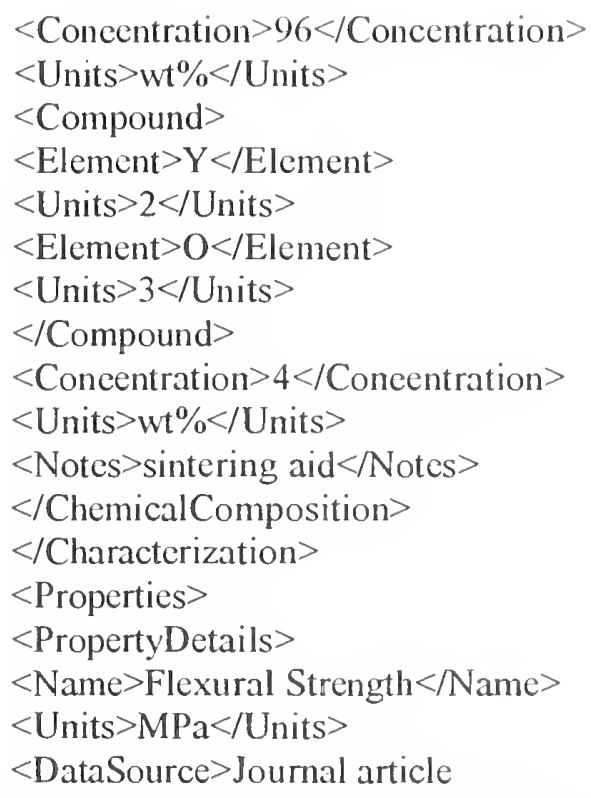

Title - Reliable Ceramics for Advanced Heat Engines

Author(s) - V.K. Pujari, D.M. Tracey, M.F. Foley, N.I. Paille, P.J. Pelletier, L.C. Sales, C.A.

Willkens, and R.L. Yeckley

Publication - American Ceramic Society Bulletin

Volume - 74

Issue - 4

Year -1995

Page(s) - 86-90

Publisher - American Ceramic Society

$<$ DataSource $>$

$<$ DataType $>$ Evaluated $<$ /DataType $>$

$<$ MeasurementTechnique $>$

$<$ Name $>$ Strength tests $<$ Name $>$

$<$ Notes $>$

The authors cite V.R. Pujari et al., "Development of Improved Processing and Evaluation Methods for High Reliability Structural Ceramics for Advanced Heat Engine Applications, Phase I," final report, ORNL/Sub/89-SB182/1, NTIS Rept. No. DE93-040528, August (1993), and summarize the procedure as follows. "The cylindrical buttonhead specimens were machined to ORNL design with a gauge diameter of $6.0 \pm 0.1 \mathrm{~mm}$. ...50 $\mathrm{mm}$ diameter, $150 \mathrm{~mm}$ long specimens... were machined as many flexure bars $(3 \mathrm{~mm}$ by $4 \mathrm{~mm}$ by $50 \mathrm{~mm}$ ) for assessment of the properties across the $50-\mathrm{mm}$ section."

$<$ Notes $>$

$</$ Measurement Tcehnique $>$

$<$ Notes $>$

Cautions: Evaluated Data.

"The nonlinear character of the distribution with multiple infleetions suggests that a twoparameter Weibull fit of these data $(\operatorname{sigma}=1038 \mathrm{MPa}, \mathrm{m}=10.4)$ is inappropriate and that the multimodal nature of the data should be represented using competing risk analysis.... The important feature of the three-parameter Weibull distribution is the existence of a threshold stress below which there is zero probability of failure."

$<$ Notes $>$

$</$ PropertyDetails $>$ 
$<$ Value type $=$ "integer" $>972,561<$ Value $>$

$<$ Parameters $>$

$<$ Name $>$ Test Temperature $<$ Name $>$

$<$ Value type $=$ "integer" $>23,1370</$ Value $>$

$<$ Units $>$ \&\#176; C </Units $>$

$</$ Parameters $>$

$<$ PropertyDetails $>$

$<$ Name $>$ Tensile Strength $<$ Name $>$

$<$ Units $>\mathrm{MPa}</$ Units $>$

$<$ DataSource $>$ Journal article

Title - Reliable Ceramics for Advanced Heat Engines

Author(s) - V.K. Pujari, D.M. Tracey, M.F. Foley, N.I. Paille, P.J. Pelletier, L.C. Sales, C.A.

Willkens, and R.L. Yeckley

Publication - American Ceramic Society Bulletin

Volume - 74

Issue -4

Year - 1995

Page(s) - 86-90

Publisher - American Ceramic Society

$</$ DataSource $>$

$<$ DataType $>$ Evaluated $<$ DataType $>$

$<$ MeasurementTechnique $>$

$<$ Name $>$ Strength tests $<$ Name $>$

$<$ Notes $>$

The authors cite V.R. Pujari et al., "Development of Improved Processing and Evaluation Methods for High Reliability Structural Ceramics for Advanced Heat Engine Applications, Phase I," final report, ORNL/Sub/89-SB182/1, NTIS Rept. No. DE93-040528, August (1993), and summarize the procedure as follows. "The cylindrical buttonhead specimens were machined to ORNL design with a gauge diameter of $6.0 \pm 0.1 \mathrm{~mm}$...50 $\mathrm{mm}$ diameter, $150 \mathrm{~mm}$ long specimens... were machined as many flexure bars $(3 \mathrm{~mm}$ by $4 \mathrm{~mm}$ by $50 \mathrm{~mm}$ ) for assessment of the properties across the 50-mm section."

$<$ Notes $>$

$<$ /MeasurementTechnique $>$

$<$ Notes $>$

Cautions: Evaluated Data.

"The nonlinear character of the distribution with multiple inflections suggests that a twoparameter Weibull fit of these data $($ sigma $=1038 \mathrm{MPa}, m=10.4)$ is inappropriate and that the multimodal nature of the data should be represented using competing risk analysis. ... The important feature of the three-parameter Weibull distribution is the existence of a threshold stress below which there is zero probability of failure."

$<$ Notes $>$

$</$ PropertyDetails $>$

$<$ Value type $="$ integer" $>997,396</$ Value $>$

$<$ Parameters $>$

$<$ Name $>$ Test Temperature $<$ Name $>$

$<$ Value type $=$ "integer" $>23,1370</$ Value $>$

$<$ Units $>\& \# 176$;C</Units $>$

$<$ Name $>$ Range of Strengths $<$ Name $>$

$<$ Value type $=$ "text" $>540-1237,344-452<$ /Value $>$

$<$ Units $>\mathrm{MPa}</$ Units $>$ 
$<$ /Parameters $>$

$<$ PropertyDetails $>$

$<$ Name $>$ Weibull Strength $<$ Name $>$

$<$ Units $>\mathrm{MPa}</$ Units $>$

$<$ DataSource $>$ Journal article

Title - Reliablc Ceramics for Advanced Heat Engines

Author(s) - V.K. Pujari, D.M. Tracey, M.F. Foley, N.I. Paille, P.J. Pelletier, L.C. Sales, C.A.

Willkens, and R.L. Yeckley

Publication - American Ceramic Society Bulletin

Volume - 74

Issue - 4

Year -1995

Page(s) - 86-90

Publisher - American Ceramic Society

$</$ DataSourcc $>$

$<$ DataType $>$ Evaluated $<$ /DataType $>$

$<$ Measurcment Technique $>$

$<$ Name $>$ Strength tests $<$ Name $>$

$<$ Notes $>$

The authors cite V.R. Pujari et al., "Development of Improved Processing and Evaluation Methods for High Reliability Structural Ceramics for Advanced Heat Engine Applications, Phasc I," final report,

ORNL/Sub/89-SB182/1, NTIS Rept. No. DE93-040528, August (1993), and summarize the procedure as follows. "The cylindrical buttonhead specimens were machined to ORNL design with a gauge diameter of $6.0 \pm 0.1 \mathrm{~mm} . .50 \mathrm{~mm}$ diameter, $150 \mathrm{~mm}$ long specimens... were machined as many flexure bars $(3 \mathrm{~mm}$ by $4 \mathrm{~mm}$ by $50 \mathrm{~mm}$ ) for assessment of the properties across the 50-mm section."

$<$ Notes $>$

$<$ /MeasurementTechnique $>$

$<$ Notes $>$

Cautions: Evaluated Data.

"The nonlinear character of the distribution with multiple inflections suggests that a twoparameter Weibull fit of these data $(\operatorname{sigma}=1038 \mathrm{MPa}, \mathrm{m}=10.4)$ is inappropriate and that the multimodal nature of the data should be represented using competing risk analysis... The important feature of the thrce-parameter Wcibull distribution is the existence of a threshold stress below which therc is zero probability of failure."

$<$ Notes $>$

$</$ PropertyDetails $>$

$<$ Value type $=$ "integer" $>1109</$ Value $>$

$<$ Parameters $>$

$<$ Name $>$ Test Temperature $<$ Name $>$

$<$ Value type $=$ "integer" $>23<$ Value $>$

$<$ Units $>\& \# 176 ; \mathrm{C}</$ Units $>$

$<$ Name $>$ Weibull Modulus $<$ Name $>$

$<$ Value type $=$ "integer" $>4</$ Value $>$

$<$ Units $>$ none $</$ Units $>$

$<$ Name $>$ Threshold Strength $<$ Name $>$

$<$ Value type $=$ "integer" $>665</$ Value $>$

$<$ Units $>\mathrm{MPa}</$ Units $>$

$</$ Parameters $>$

$<$ PropertyDetails $>$ 
$<$ Name $>$ Weibull Modulus $<$ Name $>$

$<$ Units $>$ none $</$ Units $>$

$<$ DataSource $>$ Journal article

Title - Reliable Ceramics for Advanced Heat Engines

Author(s) - V.K. Pujari, D.M. Tracey, M.F. Foley, N.I. Paille, P.J. Pelletier, L.C. Sales, C.A.

Willkens, and R.L. Yeckley

Publication - American Ceramic Society Bulletin

Volume - 74

Issue - 4

Year -1995

Page(s) - 86-90

Publisher - American Ceramic Society

$</$ DataSource $>$

$<$ DataType $>$ Evaluated $<$ DataTypc $>$

$<$ Measurement Technique $>$

$<$ Name $>$ Strength tests $<$ /Name $>$

$<$ Notes $>$

The authors cite V.R. Pujari et al., "Development of Improved Processing and Evaluation Methods for High Reliability Structural Ceramics for Advanced Heat Engine Applications, Phase I," final report, ORNL/Sub/89-SB182/1, NTIS Rept. No. DE93-040528, August (1993), and summarize the procedure as follows. "The cylindrical buttonhead specimens were machined to ORNL design with a gauge diameter of $6.0 \pm 0.1 \mathrm{~mm} . .50 \mathrm{~mm}$ diameter, $150 \mathrm{~mm}$ long specimens ... were machined as many flexure bars $(3 \mathrm{~mm}$ by $4 \mathrm{~mm}$ by $50 \mathrm{~mm}$ ) for assessment of the properties across the 50-mm section."

$<$ Notes $>$

$<$ MeasurementTechnique $>$

$<$ Notes $>$

Cautions: Evaluated Data.

"The nonlinear character of the distribution with multiple inflections suggests that a twoparameter Weibull fit of these data (sigma $=1038 \mathrm{MPa}, \mathrm{m}=10.4$ ) is inappropriate and that the multimodal nature of the data should be represented using competing risk analysis. ... The important feature of the three-parameter Weibull distribution is the existence of a threshold stress below which there is zero probability of failure."

$<$ /Notes $>$

$</$ PropertyDetails $>$

$<$ Value type $=$ "text" $>4,---,---<$ Value $>$

$<$ Parameters $>$

$<$ Name $>$ Stress Mode $<$ /Name $>$

$<$ Value type $=$ "text" $>$ Tensile,Flexural,Flexural $</$ Value $>$

$<$ Units $>$ none $</$ Units $>$

$<$ Name $>$ Test Temperature $<$ /Name $>$

$<$ Value type $=$ "integer" $>23,23,1370<$ Nalue $>$

$<$ Units $>$ \&\#176; C</Units $>$

$<$ Name $>$ Threshold Strength $</$ Name $>$

$<$ Value type $=$ "integer" $>665,653,517</$ Value $>$

$<$ Units $>\mathrm{MPa}</$ Units $>$

$<$ Name $>$ Weibull Strength $<$ /Name $>$

$<$ Value type="text" $>1109,---,--</$ Value $>$

$<$ Units $>\mathrm{MPa}</$ Units $>$

$<$ /Parameters $>$ 
$</$ Properties $>$

$<$ /BulkDetails $>$

$<$ /Material $>$

$<$ MatML_Doc $>$ 
Example 2: Aluminum alloy from a printed handbook

Source: Generously provided by F. Cverna of ASM International and J.G. Kaufman of the Aluminum Association from Properties of Aluminum Alloys, p. 291, ASM International, Materials Park, Ohio, ISBN: 0-87170-632-6, 1999.

$<? \mathrm{xml}$ version $=" 1.0 " ?>$

$<$ !--DOCTYPE MatML_Doc SYSTEM "MatMLv20.dtd"-->

$<$ MatML Doc $>$

$<$ Material $>$

$<$ BulkDetails $>$

$<$ Name $>$ aluminum alloy $<$ Name $>$

$<$ Class $>$ metal $</$ Class $>$

$<$ Specification $>1350</$ Specification $>$

$<$ Form $>$ Rolled rod and shapes; smooth specimens $</$ Form $>$

$<$ Processing $>$

$<$ Name $>$ Temper $<$ Name $>$

$<$ Notes $>$ H1 $8<$ Notes $>$

$</$ Processing $>$

$<$ Properties $>$

$<$ PropertyDetails $>$

$<$ Name $>$ Axial-Stress Fatigue Strength $<$ /Name $>$

$<$ Units $>$ ksi $</$ Units $>$

$<$ DataSource $>$

"Properties of aluminum alloys : tensile, creep, and fatigue data at high and low temperatures" / edited by J. Gilbert Kaufman.

$</$ DataSource $>$

$<$ DataType $>$ Handbook $<$ DataType $>$

$<$ Notes $>$ Plus $(+)$ indicates tension; minus $(-)$ indicates compression. $<$ Notes $>$

$<$ /PropertyDetails $>$

$<$ Value type $=$ "float" $>+23,+17,+15,+14.5,+14.5</$ Value $>$

$<$ Parameters $>$

$<$ Name $>$ Stress Ratio $(\mathrm{R})<$ Name $>$

$<$ Value type $=$ "integer" $>0,0,0,0,0</$ Value $>$

$<$ Units $>$ none $</$ Units $>$

$<$ Notes $>$ Stress Ratio $(R)=($ minimum stress $) /($ maximum stress $)</$ Notes $>$

$<$ Name $>$ Number of Samples $<$ Name $>$

$<$ Value type $=$ "integer" $>1,1,1,1,1</$ Value $>$

$<$ Units $>$ none $</$ Units $>$

$<$ Name $>$ Number of Cycles $<$ Name $>$

$<$ Value type $=$ "integer" $>10^{\wedge} 5,10^{\wedge} 6,10^{\wedge} 7,10^{\wedge} 8,5 \times 10^{\wedge} 8</$ Value $>$

$<$ Units $>$ none $</$ Units $>$

$</$ Parameters $>$

$<$ PropertyDetails $>$

$<$ Name $>$ Axial-Stress Fatigue Strength $<$ Name $>$

$<$ Units $>\mathrm{MPa}<$ /Units $>$

$<$ DataSource $>$

"Properties of aluminum alloys : tensile, creep, and fatigue data at high and low temperatures" /

edited by J. Gilbert Kaufman.

$</$ DataSource $>$

$<$ DataType $>$ Handbook $<$ DataType $>$

$<$ Notes $>$ Plus $(+)$ indicates tension; minus (-) indicates compression. $<$ Notes $>$ 
$</$ PropertyDetails $>$

$<$ Value type $=$ "integer" $>+160,+115,+105,+100,+100</$ Value $>$

$<$ Parameters $>$

$<$ Name $>$ Stress Ratio $(\mathrm{R})<$ Name $>$

$<$ Value type $=$ "integer" $>0,0,0,0,0</$ Value $>$

$<$ Units $>$ none $</$ Units $>$

$<$ Name $>$ Number of Samples $<$ Name $>$

$<$ Value type $=$ "integer" $>1,1,1,1,1</$ Value $>$

$<$ Units $>$ none $</$ Units $>$

$<$ Name $>$ Number of Cyeles $</$ Name $>$

$<$ Value type $="$ integer" $>10^{\wedge} 5,10^{\wedge} 6,10^{\wedge} 7,10^{\wedge} 8,5 \times 10^{\wedge} 8<\sim$ Value $>$

$<$ Units $>$ none $</$ Units $>$

$<$ /Parameters $>$

$<$ PropertyDetails $>$

$<$ Name $>$ Axial-Stress Fatigue Strength $<$ Name $>$

$<$ Units $>$ ksi $</$ Units $>$

$<$ DataSource $>$

"Properties of aluminum alloys : tensile, creep, and fatigue data at high and low temperatures" / edited by J. Gilbert Kaufman.

$<$ /DataSouree $>$

$<$ DataType $>$ Handbook $<$ /DataType $>$

$<$ Notes $>$ Plus $(+)$ indieates tension; minus $(-)$ indieates eompression. $<$ Notes $>$

$</$ PropertyDetails $>$

$<$ Value type $=$ "float" $>+11.5,+8.5,+7,+6.5,+6.5<$ Value $>$

$<$ Parameters $>$

$<$ Name $>$ Stress Ratio $(\mathrm{R})</$ Name $>$

$<$ Value type $=$ "integer" $>-1,-1,-1,-\mathrm{I},-\mathrm{I}</$ Value $>$

$<$ Units $>$ none $</$ Units $>$

$<$ Name $>$ Number of Samples $<$ Name $>$

$<$ Value type $=$ "integer" $>1,1,1,1,1</$ Value $>$

$<$ Units $>$ none $</$ Units $>$

$<$ Name $>$ Number of Cyeles $</$ Name $>$

$<$ Value type $="$ integer" $>10^{\wedge} 5,10^{\wedge} 6,10^{\wedge} 7,10^{\wedge} 8,5 \times 10^{\wedge} 8<$ Value $>$

$<$ Units $>$ none $</$ Units $>$

$</$ Parameters $>$

$<$ PropertyDetails $>$

$<$ Name $>$ Axial-Stress Fatigue Strength $</$ Name $>$

$<$ Units $>\mathrm{MPa}</$ Units $>$

$<$ DataSouree $>$

"Properties of aluminum alloys : tensile, ereep, and fatigue data at high and low temperatures" / edited by J. Gilbert Kaufman.

$<$ /DataSouree $>$

$<$ DataType $>$ Handbook $<$ /Data'Type $>$

$<$ Notes $>$ Plus ( + ) indieates tension; minus (-) indieates eompression $</$ Notes $>$

$</$ PropertyDetails $>$

$<$ Value type $=$ "float" $>+80,+59,+48,+45,+45</$ Value $>$

$<$ Parameters $>$

$<$ Name $>$ Stress Ratio $(\mathrm{R})</$ Name $>$

$<$ Value type $=$ "integer" $>-1,-1,-1,-1,-1</$ Value $>$

$<$ Units $>$ none $</$ Units $>$

$<$ Name $>$ Number of Samples $</$ Name $>$ 
$<$ Value type $=$ "integer" $>1,1,1,1,1<$ /Value $>$

$<$ Units $>$ none $</$ Units $>$

$<$ Name $>$ Number of Cycles $<$ Name $>$

$<$ Value type $="$ integer" $>10^{\wedge} 5,10^{\wedge} 6,10^{\wedge} 7,10^{\wedge} 8,5 \times 10^{\wedge} 8</$ Value $>$

$<$ Units $>$ none $</$ Units $>$

$</$ Parameters $>$

$</$ Properties $>$

$<$ /BulkDetails $>$

$<$ Terms $>$

$<$ termEntry $>$

$<$ term $>$ H $18</$ term $>$

$<$ definition $>$

"H18" is a code from The Aluminum Association Temper Designation System.

The $\mathrm{H}$ is defined as "strain-hardened (wrought products only). The 1 applies to products that are strain-hardened to obtain the desired strength without supplementary thermal treatment. The 8 indicates the degree of strain-hardening and is assigned to the hardest tempers normally produced.

$</$ definition $>$

$</$ termEntry $>$

$<$ termEntry $>$

$<$ term $>1350</$ term $>$

$<$ definition $>$

"1350" is a code from The Aluminum Association Alloy Designation System. The first digit of the code represents the principal alloying constitutent(s). The second digit indicates variations of the initial alloy. The third and fourth digits indicate individual alloy variations (the numbers have no significance but are unique). 1350 is an alloy that is pure $\mathrm{AL}(99.00 \%$ or greater). For further details, contact The Aluminum Association, 900 19th Street, N.W., Washington, D.C. 20006.

$</$ definition $>$

$</$ termEntry $>$

$</$ Terms $>$

$<$ /Material $>$

$</$ MatML_Doc $>$ 
Example 3: Steel with TiC coating from a journal article

Source: A. Agarwal and N.B. Dahotre, "Pulsed Electrode Surfacing of Steel With TiC Coating: Microstructure and Wear Properties," ASM Journal of Materials Engineering and Performance, Vol. 8, No. 4, pp. 479-486, 1999.

$<$ ?xml version=" $1.0 " ?>$

$<$ !--DOCTYPE MatML_Doc SYSTEM "MatMLv20.dtd"-->

$<$ MatML_Doc $>$

$<$ Material $>$

$<$ BulkDetails $>$

$<$ Name $>$ TiC coated AISI 1018 steel $<$ Name $>$

$<$ Class $>$ composite $</$ Class $>$

$<$ Subclass $>$ ceramic coating on metal substrate $</$ Subclass $>$

$<$ Form $>$ coupon $</$ Form $>$

$<$ Properties $>$

$<$ PropertyDetails $>$

$<$ Name $>$ Wear (Weight Loss Analysis) $<$ Name $>$

$<$ Units $>$ g $</$ Units $>$

$<$ DataSource $>$

A. Agarwal and N.B. Dahotre, "Pulse Electrode Surfacing of Steel with TiC Coating:

Microstructure and Wear Properties," ASM Journal of Materials Engineering and Performance,

Vol. 8, No. 4, pp. 479-486, 1999

$</$ DataSource $>$

$<$ MeasurementTechnique $>$

$<$ Name $>$ Block-on-disk tribometer $<$ Name $>$

$<$ Notes $>$

"Coated coupons of dimension $25 \times 25 \mathrm{~mm}$ were tested for dry sliding wear against a hardened steel ring rotating at a linear speed of $270 \mathrm{~m} / \mathrm{min}$. Weight loss measurements were made after successive 2

min. The dry sliding wear test was conducted for 10 min with an applied normal load of $2 \mathrm{~kg}$."

$<$ Notes $>$

$<$ MeasurementTechnique $>$

$<$ Notes $>$

Data were digitized from Fig. 9. The reported unit, "gm", is interpreted to mean "g", grams.

$<$ Notes $>$

$<$ PropertyDetails $>$

$<$ Value type $=$ "float" $>.0011, .0018, .0023, .0027, .0029</$ Value $>$

$<$ Parameters $>$

$<$ Name $>$ Time $<$ Name $>$

$<$ Value type $=$ "integer" $>2,4,6,8,10<$ Value $>$

$<$ Units $>$ minutes $</$ Units $>$

$<$ Name $>$ Sliding Speed (Steel Ring) $<$ Name $>$

$<$ Value type $=$ "integer" $>270,270,270,270,270<$ /Value $>$

$<$ Units $>$ m/minute $</$ Units $>$

$<$ Notes $>$ See details in MeasurementTechnique Notes. $\quad<$ /Notes $>$

$<$ Name $>$ Applied Normal Load $<$ Name $>$

$<$ Value type $=$ "integer" $>2,2,2,2,2<$ /Value $>$

$<$ Units $>$ kg $</$ Units $>$

$</$ Parameters $>$

$<$ PropertyDetails $>$

$<$ Name $>$ Coefficient of Friction $<$ /Name $>$ 
$<$ Units $>$ none $</$ Units $>$

$<$ DataSource $>$ See DataSource under Wear (Weight Loss Analysis) $</$ DataSource $>$

$<$ MeasurementTechnique $>$

$<$ Name $>$ Block-on-disk tribometer $<$ Name $>$

$<$ Notes $>$

"The coefficient of friction $(\mu)$ was also recorded simultaneously by an interface computer, which acquired data in the form of electrical output power of the motor. Even though data were recorded at a frequency of $1 \mathrm{~Hz}$ for a total test time of $10 \mathrm{~min}$, an average of 10 successive points was taken for computing the coefficient of friction, $\mu$....the coefficient of friction is calculated by measuring the changes in voltage and

current in the electrical circuit of the motor driving the block-on-ring tribometer during loading..."

$<$ Notes $>$

$<$ Measurement'Technique $>$

$</$ PropertyDetails $>$

$<$ Value type $=$ "float" $>0.58</$ Value $>$

$<$ /Properties $>$

$</$ BulkDetails $>$

$<$ ComponentDetails $>$

$<$ Name $>$ steel $<$ Name $>$

$<$ Class $>$ metal $</$ Class $>$

$<$ Specification authority="American Iron and Steel Institute" $>$ AISI $1018</$ Specification $>$

$<$ Form $>$ coupon $</$ Form $>$

$<$ Processing $>$

$<$ Name $>$ Mechanical polishing $<$ Name $>$

$<$ Notes $>\quad$ The coupons were mechanically polished on emery paper of grit size

$240 .<$ Notes $>$

$<$ Name $>$ Rinsing $<$ Name $>$

$<$ Notes $>\quad$ After polishing, the coupons were rinsed in acetone. $<$ Notes $>$

$<$ Name $>$ Coating $<$ Name $>$

$<$ Notes $>$

"A sintered electrode of $\mathrm{TiC}$ was used to deposit a coating on these steel coupons. The TiC electrode had 3 to $5 \mathrm{wt} \% \mathrm{Ni}$ and 1 to $3 \mathrm{wt} \% \mathrm{Fe}$ as binder. Deposition was carried out using a handheld gun in air at room temperature. Pulsed electrode deposition was carried out at a voltage of $50 \mathrm{~V}$ and spark time of $10 \mu \mathrm{s}$. The discharge capacitance used for the PES process was $450 \mu \mathrm{F}$ with a current of $25 \mathrm{~A} . "$

$<$ Notes $>$

$</$ Processing $>$

$<$ Geometry $>$

$<$ Shape $>$ square $</$ Shape $>$

$<$ Dimensions $>25 \mathrm{mmn} \times 25 \mathrm{~mm}</$ Dimensions $>$

$</$ Geometry $>$

$<$ Properties $>$

$<$ PropertyDetails $>$

$<$ Name $>$ Wear (Weight Loss Analysis) $<$ Name $>$

$<$ Units $>$ g $</$ Units $>$

$<$ DataSource $>$ See DataSource under Wear (Weight Loss Analysis) in BulkDetails. </DataSource $>$

$<$ MeasurementTechnique>

$<$ Name $>$ Block-on-disk tribometer $<$ /Name $>$

$<$ /MeasurementTechnique $>$

$<$ Notes $>\quad$ See Notes under Wear (Weight Loss Analysis) in BulkDetails. $<$ /Notes $>$ 
$</$ PropertyDetails $>$

$<$ Value type $=$ "float" $>.0019, .0036, .0057, .0073, .0090<$ /Value $>$

$<$ Parameters $>$

$<$ Name $>$ Time $<$ Name $>$

$<$ Value type $=$ "integer" $>2,4,6,8,10<$ Value $>$

$<$ Units $>$ minutes $</$ Units $>$

$<$ Name $>$ Sliding Speed (Steel Ring) $<$ Name $>$

$<$ Value type $=$ "integer" $>270,270,270,270,270<$ /Value $>$

$<$ Units $>$ m/minute $</$ Units $>$

$<$ Name $>$ Applied Normal Load $<$ Name $>$

$<$ Value type $=$ "integer" $>2,2,2,2,2<$ Value $>$

$<$ Units $>$ kg $</$ Units $>$

$</$ Parameters $>$

$<$ PropertyDetails $>$

$<$ Name $>$ Microhardness $<$ /Name $>$

$<$ Units $>\mathrm{kg} / \mathrm{mm}^{\wedge} 2</$ Units $>$

$<$ Measurement Technique $>$

$<$ Name $>$ Knoop Indentation $</$ Name $>$

$<$ Notes $>$

"Microhardness measurements were performed on a Buchler Micromet II microhardness tester

using a Knoop indenter with normal load of $200 \mathrm{~g}$ applied for $15 \mathrm{~s}$."

$<$ Notes $>$

$</$ MeasurementTechnique $>$

$</$ PropertyDetails $>$

$<$ Value type $="$ text" $>172 \pm 12</$ Valuc $>$

$</$ Propertics $>$

$<$ Associations $>$

$<$ Associate $>$ titanium carbide coating $</$ Associate $>$

$<$ Relationship $>$ substrate $</$ Relationship $>$

$<$ Associations $>$

$<$ Name $>$ titanium carbide coating $</$ Name $>$

$<$ Class $>$ carbide $</$ Class $>$

$<$ Subclass $>$ monocarbide $</$ Subclass $>$

$<$ Characterization $>$

$<$ Formula $>$ TiC $\times$ Fe $</$ Formula $>$

$<$ PhaseComposition $>$

$<$ Name $>$ TiC $<$ Name $>$

$<$ Concentration type $=$ "text" $>$ Not reported $</$ Concentration $>$

$<$ Units $>$ Not applicable $</$ Units $>$

$<$ Name $>$ Ti $<$ /Name $>$

$<$ Concentration type $=$ "text" $>5-25</$ Concentration $>$

$<$ Units $>$ wt $\%</$ Units $>$

$<$ Name $>$ Fe-C (austenite) $<$ Name $>$

$<$ Concentration type $=$ "text" $>$ Not reported $</$ Concentration $>$

$<$ Units $>$ Not applicable $</$ Units $>$

$<$ Name $>$ Fe (ferrite) $<$ Name $>$

$<$ Concentration type $=$ "text" $>$ Not reported $</$ Concentration $>$

$<$ Units $>$ Not applicable $</$ Units $>$

$<$ Name $>$ FeTi $<$ Namc $>$

$<$ Concentration type $=$ "text" $>$ Not reported $</$ Concentration $>$

$<$ Units $>$ Not applicable $</$ Units $>$ 
$<$ Qualifier $>$ Possible $<$ Q Qualifier $>$

$</$ PhaseComposition $>$

$</$ Characterization $>$

$<$ Properties $>$

$<$ PropertyDetails $>$

$<$ Name $>$ Microhardness $<$ Name $>$

$<$ Units $>\mathrm{kg} / \mathrm{mm}^{\wedge} 2</$ Units $>$

$<$ Measurement Technique $>$

$<$ Name $>$ Knoop Indentation $<$ Name $>$

$<$ Notes $>\quad$ See MeasurementTechnique Notes for Microhardness in the steel

component. $<$ Notes $>$

$<$ /MeasurementTechnique $>$

$<$ PropertyDetails $>$

$<$ Value type $=$ "text" $>1235 \pm 86</$ Value $>$

$<$ Properties $>$

$<$ Associations $>$

$<$ Associate $>$ AISI 1018 steel</Associate $>$

$<$ Relationship $>$ coating $</$ Relationship $>$

$</$ Associations $>$

$<$ Name $>$ Heat Affected Zone $($ HAZ $)<$ Name $>$

$<$ Properties $>$

$<$ PropertyDetails $>$

$<$ Name $>$ Microhardness $<$ Name $>$

$<$ Units $>\mathrm{kg} / \mathrm{mm}^{\wedge} 2</$ Units $>$

$<$ MeasurementTechnique $>$

$<$ Name $>$ Knoop Indentation $<$ Name $>$

$<$ Notes $>\quad$ See MeasurementTechnique Notes for Microhardness in the steel

component. $<$ Notes $>$

$<$ /MeasurementTechnique $>$

$</$ PropertyDetails $>$

$<$ Value type $=$ "text" $>352 \pm 32<$ Value $>$

$</$ Properties $>$

$<$ Notes $>$ Martensitic zone $<$ Notes $>$

$</$ ComponentDetails $>$

$<$ /Material $>$

$</$ MatML_Doc $>$ 

